\title{
Characterization of Satisfactory Mechanisms for the Revelation of Preferences for Public Goods
}

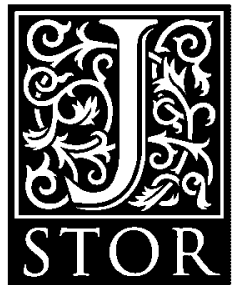

\author{
Jerry Green, Jean-Jacques Laffont \\ Econometrica, Volume 45, Issue 2 (Mar., 1977), 427-438.
}

Stable URL:

http://links.jstor.org/sici?sici=0012-9682\%28197703\%2945\%3A2\%3C427\%3ACOSMFT\%3E2.0.CO\%3B2-Q

Your use of the JSTOR archive indicates your acceptance of JSTOR's Terms and Conditions of Use, available at http://www.jstor.org/about/terms.html. JSTOR's Terms and Conditions of Use provides, in part, that unless you have obtained prior permission, you may not download an entire issue of a journal or multiple copies of articles, and you may use content in the JSTOR archive only for your personal, non-commercial use.

Each copy of any part of a JSTOR transmission must contain the same copyright notice that appears on the screen or printed page of such transmission.

Econometrica is published by The Econometric Society. Please contact the publisher for further permissions regarding the use of this work. Publisher contact information may be obtained at http://www.jstor.org/journals/econosoc.html.

Econometrica

(C1977 The Econometric Society

JSTOR and the JSTOR logo are trademarks of JSTOR, and are Registered in the U.S. Patent and Trademark Office. For more information on JSTOR contact jstor-info@umich.edu.

(C2002 JSTOR

http://www.jstor.org/ Tue Jan 29 18:48:13 2002 


\title{
CHARACTERIZATION OF SATISFACTORY MECHANISMS FOR THE REVELATION OF PREFERENCES FOR PUBLIC GOODS
}

\author{
By JeRry Green AND JEAN-JACQues LAFFOnT ${ }^{1}$
}

\begin{abstract}
Social decision mechanisms that admit dominant strategies and result in Pareto optima are characterized by the class of mechanisms proposed by Groves. The concept of decision mechanisms is generalized and the characterization is shown to extend to these cases.
\end{abstract}

PRoblems CONNECTED With social decision processes in models with public goods have troubled economists for some time. Recently a negative result of Gibbard [2] and Satterthwaite [6] precluded the possibility of finding nondictatorial deterministic mechanisms for choosing social states which have the property that individuals believe it to be impossible to manipulate the mechanism to their own advantage. They may, in particular, reveal preferences other than their own, and the resulting social choice may then be distorted away from the Pareto optimum relative to their true tastes.

Gibbard and Satterthwaite's requirements are quite strong. In particular, arbitrary individual preferences are allowed. In a more specialized context, where the decision concerns the level of public goods and monetary transfers among individuals, Groves [3] and Groves and Loeb [5] assumed that preferences are monotonic in income and that the willingness-to-pay for alternative levels of the public good is independent of income. In such environments they found a class of mechanisms with the properties that stating one's true preferences is a dominant strategy for each individual and that a Pareto optimum is selected.

In this paper, we show that the mechanisms proposed by Groves and Loeb are the only ones which have these desirable characteristics. This result enables us to concentrate the search for optimal mechanisms within this class and to use criteria other than individual incentive compatibility to distinguish among these. We have pursued this direction in other papers. In addition, we show that well-defined mechanisms which select Pareto optimal outcomes (referred to as satisfactory mechanisms), independently of the question of truthful revelation, are essentially isomorphic to the mechanisms proposed by Groves.

Section 1 sets forth notation and the basic definitions. Section 2 provides, in a world of separable utility functions, a complete characterization of satisfactory mechanisms. In an appendix we show the non-existence of satisfactory mechanisms when nonseparable utility functions are allowed.

\footnotetext{
${ }^{1}$ We are grateful to the referee for his comments. This research was supported under National Science Foundation grant SOC71-03803 to Harvard University and GS 40104 to the Institute for Mathematical Studies in the Social Sciences at Stanford University.
} 


\section{DEFINITIONS}

\section{A. Public Projects}

We are concerned with the realization of a package of public projects in an economy with a unique private good (which can be thought of as money) and $N$ agents. The only restriction imposed in the analysis on the public projects is that the set of available public project packages be a compact set $\mathscr{K}$ in a topological space. Examples include: (i) a fixed-size unique public project; then $\mathscr{K}=\{0,1\}$ where 1 represents the realization of the project and 0 the nonrealization; (ii) a variable-size unique public project; then the allowable size should belong to a bounded closed subset of $R$; for example, $\mathscr{K}=0 \cup\left[K_{1}, K_{2}\right]$ if a minimal size is required technologically or $\mathscr{K}=\left\{0, K_{1}, \ldots, K_{L}\right\}$, if indivisibilities exist; (iii) a set of $L$ variable size public projects; then, for example, $\mathscr{K}=\Pi_{l=1}^{L}\left[0, K_{l}\right]$; (iv) a compact set of functions (e.g., tax laws); then, for example, $\mathscr{K}=\mathscr{C}[0,1]$, set of continuous real functions on the interval $[0,1]$, and so forth.

Let $K$ be an element of $\mathscr{K}$.

\section{B. Transfers, Utility Functions, and Valuation Functions}

Simultaneously with public goods, we consider monetary transfers $t_{i}, i=$ $1, \ldots, N$, so that starting from an initial position the gain in utility of agent $i$ due to a program of public projects and transfers is: $u_{i}\left(K, t_{i}\right)$ defined on $\mathscr{K} \times R$.

Definition 1: The utility function $u_{i}$ is said to be (additively) separable iff

$$
u_{i}\left(K, t_{i}\right)=v_{i}(K)+t_{i} \text {. }
$$

The function $v_{i}(\cdot)$, which is considered net of the imputed costs (defined ex ante) of the project, is referred to as the valuation function of agent $i$. The assumption of separability amounts to absence of income effect in the evaluation of public goods.

The paper, with the exception of the appendix, is concerned only with separable utility functions; most definitions apply to this case with trivial generalizations for the general case.

\section{Mechanisms}

In order to solve the free rider problem we subject the $N$ agents to a game which is played according to the following rules (or mechanisms):

Let $S_{i}, i=1, \ldots, N$, be the strategy space of agent $i$, and let $S=\prod_{i=1}^{N} S_{i}$. A play of the game is an element $s=\left(s_{1}, \ldots, s_{N}\right) \in S$.

The outcomes of the game are defined by two functions: (i) For each play, a decision function $d(\cdot)$ from $S$ into $\mathscr{K}$ specifies a public project decision. (ii) For each play, a transfer rule $t(\cdot)=\left[t_{1}(\cdot), \ldots, t_{N}(\cdot)\right]$ from $S$ into $R^{N}$ specifies a program of transfers.

Let $f(\cdot)=[d(\cdot), t(\cdot)]$. 
The game can be played with different levels of information concerning the actions of the other players. If the actions of the other players are known, the outcome of the game may be a Nash equilibrium. If they are unknown, the agents may play a maximin strategy or maximize their expected utility with given subjective probability distributions over the actions of the others. In all the games considered in this paper, the optimal strategy is a dominant strategy (i.e., is optimal for any action of the other players); in this case, all these behavioral postulates regarding the players in the game are equivalent.

We introduce now several sets of rules of the game.

Definition 2: A mechanism, $M=(S, f)$, is a set of strategy spaces $S_{i}, i=$ $1, \ldots, N$, and a function $f(\cdot)=\left[d(\cdot), t_{1}(\cdot), \ldots, t_{N}(\cdot)\right]$ from $\Pi_{i=1}^{N} S_{i}=S$ into $\mathscr{K} \times R^{N}$ such that for a play $s:$ (i) the accepted project is $d(s)$, and (ii) the transfer to agent $i$ is $t_{i}(s)$ for $i=1, \ldots, N$.

DEFINITION 3: A revelation mechanism, $R M=\{V, f\}$, is a mechanism for which a strategy is a valuation function of the public project and a strategy space $S_{i}=V_{i}^{2}$ is a space of allowable valuation functions.

In a revelation mechanism, the question to the agent is: what is your valuation function? Clearly, such a mechanism can be used only if agents have separable utility functions. Only then is it meaningful to ask for the evaluation of a public project independently of the specification of the transfer. We denote by $w_{i}(\cdot)$ the answered valuation function which may be different from the true one $v_{i}(\cdot)$. Let $w(\cdot)=\left[w_{1}(\cdot), \ldots, w_{N}(\cdot)\right]$.

Definition 4: A direct revelation mechanism, $D R M=\{V, f\}$, is a revelation mechanism such that: $d(s)=d(w(\cdot)) \in\left\{K^{*} \mid K^{*} \in \mathscr{K}\right.$ and $\Sigma w_{i}\left(K^{*}\right)=\max \Sigma w_{i}(K)$, $K \in \mathscr{K}\}$.

The selection of $d(w(\cdot))$ made in the set of projects which maximize the sum of valuation functions is arbitrary; it will be also denoted by $K^{*}(w(\cdot))$. A necessary condition to use such a mechanism is that the set of maximizing projects be non-empty. Since $\mathscr{K}$ is a compact set, a sufficient condition is that the valuation functions be restricted to be upper semi-continuous (u.s.c.) on $\mathscr{K}$.

Definition 5: A Groves mechanism, $G M=\{V, f\}$, is a direct revelation mechanism with a specific transfer rule:

$$
\begin{aligned}
& \qquad t_{i}(w(\cdot))=\sum w_{-i}\left(K^{*}(w(\cdot))\right)+h_{i}\left(w_{-i}(\cdot)\right)^{3} \\
& { }^{2} V_{-i}=\prod_{\substack{j=1 \\
j \neq i}}^{N} V_{j} \text { and } V=\prod_{i=1}^{N} V_{i} . \\
& { }^{3} \text { By definition } \Sigma w_{-i}=\sum_{\substack{j=1 \\
j \neq i}}^{N} w_{j} \text { and } h_{i}\left(w_{-i}\right)=h_{i}\left(w_{1}, \ldots, w_{i-1}, w_{i+1}, \ldots, w_{N}\right) \text {. Clarke [1] intro- } \\
& \text { duced independently one element of this family for which } h_{i}\left(w_{-i}(\cdot)\right)=-\sum w_{-i}\left(K_{i}^{* *}\left(w_{-i}(\cdot)\right)\right) \text { where } \\
& K_{i}^{* *} \text { maximizes } \Sigma w_{-i}(\cdot) \text {. Vickrey }[7] \text { gave a similar mechanism for the case of private goods to avoid } \\
& \text { speculative behavior. }
\end{aligned}
$$


where $h_{i}\left(w_{-i}(\cdot)\right)$ is an arbitrary deterministic function of $w_{-i}(\cdot), i=1, \ldots, N$.

We also need a concept close to a Groves mechanism, in which transfers and decisions are not uniquely defined.

Definition 6: An extended Groves mechanism, $E G M=\{V, F\}$, is a set of allowable valuation functions $V_{i}, i=1, \ldots, N$, a decision correspondence $D(\cdot)$ from $V$ into $\mathscr{K}$ such that: each selection $d(\cdot) \in D(\cdot)$ maximizes $\Sigma w_{i}(\cdot)$; a set of arbitrary deterministic correspondences $H_{i}(\cdot)$ from $V_{-i}$ into $R, i=1, \ldots, N$, such that: $t_{i}(w(\cdot))=\Sigma w_{-i}\left(K^{*}(w(\cdot))\right)+h_{i}\left(w_{-i}(\cdot)\right)$ where $h_{i}(\cdot) \in H_{i}(\cdot), i=$ $1, \ldots, N$ and $K^{*}(w(\cdot)) \in D(w(\cdot))$.

Similarly, in an extended (direct) revelation mechanism, the decision and transfers applications are multivalued: $d(\cdot) \in D(\cdot) ; t_{i}(\cdot) \in T_{i}(\cdot), i=1, \ldots, N$. Moreover, in an extended direct revelation mechanism each $d(\cdot) \in D(\cdot)$ maximizes the sum of the answers.

\section{Properties of Mechanisms}

In designing mechanisms we are interested in different properties gathered below for convenient reference.

Given a mechanism, let $\mathscr{D}_{i}\left(v_{i}(\cdot)\right) \subset S_{i}$ be the set of dominant strategies of agent $i$ when his true valuation function is $v_{i}(\cdot)$.

Definition 7: A mechanism is said to be decisive iff

$$
\forall i, \forall v_{i}(\cdot) \in V_{i}: \mathscr{D}_{i}\left(v_{i}(\cdot)\right) \neq \varnothing .
$$

Then, $S_{i}^{\prime}=\bigcup_{v_{i}(\cdot) \in V_{i}} \mathscr{D}_{i}\left(v_{i}(\cdot)\right)$ is the set of observable strategies of agent $i$. Let $S^{\prime}=\prod_{i=1}^{N} S_{i}^{\prime}$.

Definition 8: A mechanism is said to be successful if, whenever $s=$ $\left(s_{1}, \ldots, s_{N}\right)$ and $s_{i}$ is an optimal strategy for player $i$, whose true preferences are $v_{i}$, then $d(s)$ maximizes $\Sigma v_{i}(\cdot)$.

In the particular case in which the mechanism is decisive as well as successful, so that the property of successfulness holds at all combinations of dominant strategies, we will refer to it as satisfactory. Satisfactory mechanisms are desirable because they select efficient outcomes while at the same time eliminating any strategic interaction among the agents because dominant strategies exist.

The utility of the play $s$ with the mechanism $M$ can be written (without fear of confusion) for individual $i$ as:

$$
u_{i}(s ; M)=v_{i}(d(s))+t_{i}(s) .
$$


DEFINITION 9: A revelation mechanism is strongly individually incentive compatible (s.i.i.c.), if the truth is a dominant strategy for each individual, i.e.,

$$
u_{i}\left(w_{-i}(\cdot), v_{i}(\cdot) ; R M\right) \geqslant u_{i}\left(w_{-i}(\cdot), w_{i}(\cdot) ; R M\right) \quad \forall w_{-i}(\cdot) \in V_{-i}, \forall v_{i}(\cdot) \in V_{i}
$$

In the sequel, if we have in $\mathscr{K}$ the no-action program denoted as 0 , by definition, $v_{i}(0)=0, i=1, \ldots, N$. In a revelation mechanism for which $0 \in \mathscr{K}$, we say that a strategy $w_{i}(\cdot)$ is normalized if $w_{i}(0)=0$. If all the strategies are normalized, the revelation mechanism is said to be normalized.

\section{CHARACTERIZATION OF SATISFACTORY MECHANISMS}

In a world of separable utility functions, we show first that all the s.i.i.c. successful revelation mechanisms are Groves mechanisms and, then, that all the satisfactory mechanisms are isomorphic to (extended) Groves mechanisms.

TheOREM 1 (Groves and Loeb [5]): A Groves mechanism is s.i.i.c.

Proof: For any $w_{-i}(\cdot) \in V_{-i}$ and any $w_{i}(\cdot) \in V_{i}, u_{i}\left(w_{-i}(\cdot), v_{i}(\cdot) ; G M\right)$ $-u_{i}\left(w_{-i}(\cdot), \quad w_{i}(\cdot) ; G M\right)=v_{i}\left(K^{*}\left(w_{-i}(\cdot), \quad v_{i}(\cdot)\right)\right)+\Sigma w_{-i}\left(K^{*}\left(w_{-i}(\cdot), \quad v_{i}(\cdot)\right)\right)$ $+h_{i}\left(w_{-i}(\cdot)\right)-v_{i}\left(K^{*}\left(w_{-i}(\cdot), \quad w_{i}(\cdot)\right)\right)-\Sigma w_{-i}\left(K^{*}\left(w_{-i}(\cdot), \quad w_{i}(\cdot)\right)\right)-h_{i}\left(w_{-i}(\cdot)\right)$ $=\max _{K \in \in_{i}[}\left[v_{i}(K)+\Sigma w_{-i}(K)\right]-\left[v_{i}\left(K^{*}\left(w_{-i}(\cdot), \quad w_{i}(\cdot)\right)\right)+\Sigma w_{-i}\left(K^{*}\left(w_{-1}(\cdot)\right.\right.\right.$, $\left.\left.\left.w_{i}(\cdot)\right)\right)\right] \geqslant 0$.

Q.E.D.

THEOREM 2 (Groves [4]): The set of dominant strategies for a Groves mechanism is $\left\{v_{i}(\cdot)+\alpha_{i}\right\}$ where $\alpha_{i}, i=1, \ldots, N$, are arbitrary constants. There is a unique normalized dominant strategy corresponding to $\alpha_{i}=0, i=1, \ldots, N$.

Proof: Suppose that there exists a dominant strategy, say $v_{i}^{\prime}(\cdot)$, which is not of the form $v_{i}(\cdot)+\alpha_{i}$. Then, there exist $\varepsilon>0, \alpha, K^{*} \in \mathscr{K}$, and $K^{* *} \in \mathscr{K}$ such that:

$$
v_{i}^{\prime}\left(K^{*}\right)=v_{i}\left(K^{*}\right)+\alpha
$$

and

$$
v_{i}^{\prime}\left(K^{* *}\right)=v_{i}\left(K^{* *}\right)+\alpha+\varepsilon
$$

Choose $w_{-i}(\cdot)$ u.s.c. such that:

$$
\begin{aligned}
& \sum w_{-i}\left(K^{*}\right)=-v_{i}\left(K^{*}\right)-\alpha, \\
& \sum w_{-i}\left(K^{* *}\right)=-v_{i}\left(K^{* *}\right)-\alpha-\varepsilon / 2,
\end{aligned}
$$

and

$$
\sum w_{-i}(K)=-\sup \left[\sup _{K \in \mathscr{K}} v_{i}(K), \sup _{K \in \mathscr{K}} v_{i}^{\prime}(K)\right]-\alpha-\varepsilon
$$

for $K \in \mathscr{K}, K \neq K^{*}, K \neq K^{* *}$. Clearly, the answer $v_{i}(\cdot)$ leads to the project $K^{*}$ and 
the answer $v_{i}^{\prime}(\cdot)$ leads to the project $K^{* *}$. Moreover, we have:

$$
v_{i}\left(K^{*}\right)+\sum w_{-i}\left(K^{*}\right)>v_{i}\left(K^{* *}\right)+\sum w_{-i}\left(K^{* *}\right)
$$

therefore, $v_{i}^{\prime}(\cdot)$ is not a dominant strategy, a contradiction. All the dominant strategies must then be of the form $\left\{v_{i}(\cdot)+\alpha_{i}\right\}$. From Theorem 1 , they are indeed dominant strategies.

Finally, a normalized strategy for agent $i$ is such that $w_{i}(0)=0$. Since by definition $v_{i}(0)=0, \alpha_{i}=0$ for a normalized dominant strategy.

\section{Corollary 1: A Groves mechanism is successful.}

Proof: From Theorem 2 and the definition of a Groves mechanism we know that the decision taken maximizes the sum of the valuation functions. Q.E.D.

COROLlARY 2: The set of dominant strategies for a s.i.i.c. revelation mechanism is included in the set $\left\{v_{i}(\cdot)+\alpha_{i}\right\}$ where $\alpha_{i}, i=1, \ldots, N$, are arbitrary constants. There is a unique normalized dominant strategy corresponding to $\alpha_{i}=0, i=$ $1, \ldots, N$.

Proof: As in Theorem 2, we show first that a dominant strategy for agent $i$ must be of the form $\left\{v_{i}(\cdot)+\alpha_{i}\right\} . v_{i}(\cdot)$ is a dominant strategy since the mechanism is s.i.i.c. As in Theorem 2, it is also the only normalized dominant strategy.

Q.E.D.

Definition 10: A direct revelation mechanism satisfies Property A, if and only if: for any $i=1, \ldots, N$, (i) $t_{i}(w(\cdot))$ is independent of $w_{i}(\cdot)$ at $K^{*}$, i.e., if for $w_{-i}(\cdot), w_{i}(\cdot), w_{i}^{\prime}(\cdot), K^{*}\left(w_{-i}(\cdot), w_{i}(\cdot)\right)=K^{*}\left(w_{-i}(\cdot), w_{i}^{\prime}(\cdot)\right)$, then $t_{i}\left(w_{-i}(\cdot)\right.$, $\left.w_{i}(\cdot)\right)=t_{i}\left(w_{-i}(\cdot), w_{i}^{\prime}(\cdot)\right)$; (ii) $t_{i}\left(w_{-i}(\cdot), w_{i}(\cdot)\right)-t_{i}\left(w_{-i}(\cdot), w_{i}^{\prime}(\cdot)\right)=\Sigma w_{-i}\left(K^{*}\right)$ $-\Sigma w_{-i}\left(K^{* \prime}\right)$ where $K^{*}$ maximizes $\Sigma w_{-i}(K)+w_{i}(K)$ over $\mathscr{K}$ and $K^{* \prime}$ maximizes $\Sigma w_{-i}(K)+w_{i}^{\prime}(K)$ over $\mathscr{K}$.

Lemma 1: A direct revelation mechanism is a Groves mechanism if and only if it satisfies Property $A$.

Proof: Obvious.

We are now able to prove the main characterization theorem.

TheOREM 3: A s.i.i.c. direct revelation mechanism is a Groves mechanism.

Proof: We consider in turn the negation of the two parts of Property A. If (i) fails there exist $w_{-i}(\cdot), w_{i}(\cdot)$, and $w_{i}^{\prime}(\cdot)$ which lead to the same $K^{*}$ such that

$$
t_{i}\left(w_{-i}(\cdot), w_{i}(\cdot)\right)>t_{i}\left(w_{-i}(\cdot), w_{i}^{\prime}(\cdot)\right) \text {. }
$$

Let $v_{i}(\cdot)=w_{i}^{\prime}(\cdot)$. 
Then, $t_{i}\left(w_{-i}(\cdot), w_{i}(\cdot)\right)+v_{i}\left(K^{*}\right)>t_{i}\left(w_{-i}(\cdot), v_{i}(\cdot)\right)+v_{i}\left(K^{*}\right) ; v_{i}(\cdot)$ is not a dominant strategy, a contradiction.

If (ii) fails, there exist $w_{-i}(\cdot), w_{i}(\cdot)$, and $w_{i}^{\prime}(\cdot)$ such that: $K^{*}$ maximizes $\Sigma w_{-i}(\cdot)+w_{i}(\cdot)$ over $\mathscr{K}, K^{* \prime}$ maximizes $\Sigma w_{-i}(\cdot)+w_{i}^{\prime}(\cdot)$ over $\mathscr{K}$, and $t_{i}\left(w_{-i}(\cdot)\right.$, $\left.w_{i}(\cdot)\right)-t_{i}\left(w_{-i}(\cdot), w_{i}^{\prime}(\cdot)\right)=\Sigma w_{-i}\left(K^{*}\right)-\Sigma w_{-i}\left(K^{* \prime}\right)+\varepsilon$ for some $\varepsilon>0$.

Let $\tilde{w}_{i}^{\prime}(\cdot)$ be defined as

$$
\begin{aligned}
& \tilde{w}_{i}^{\prime}\left(K^{*}\right)=-\sum w_{-i}\left(K^{*}\right), \\
& \tilde{w}_{i}^{\prime}\left(K^{* \prime}\right)=-\sum w_{-i}\left(K^{* \prime}\right)+\delta \quad \text { with } \quad 0<\delta<\varepsilon,
\end{aligned}
$$

and

$$
\tilde{w}_{i}^{\prime}(K)=-c \quad \text { for } K \neq K^{*} \text { or } K^{* \prime} \text { with } c>\max _{K \in \mathscr{K}} \sum w_{-i}(K)
$$

where $\tilde{w}_{i}^{\prime}(\cdot)$ is upper semi-continuous.

Note that $\max \tilde{w}_{i}^{\prime}(K)+\Sigma w_{-i}(K)$ is solved at $K=K^{* \prime}$ and, therefore, by the first part of the proof,

$$
t_{i}\left(w_{-i}(\cdot), w_{i}^{\prime}(\cdot)\right)=t_{i}\left(w_{-i}(\cdot), \tilde{w}_{i}^{\prime}(\cdot)\right) .
$$

We have that

$$
\begin{aligned}
t_{i}\left(w_{-i}(\cdot), w_{i}(\cdot)\right)-t_{i}\left(w_{-i}(\cdot), \tilde{w}_{i}^{\prime}(\cdot)\right) & =\sum w_{-i}\left(K^{*}\right)-\sum w_{-i}\left(K^{* \prime}\right)+\varepsilon \\
& =-\tilde{w}_{i}^{\prime}\left(K^{*}\right)+\tilde{w}_{i}^{\prime}\left(K^{* \prime}\right)+\varepsilon-\delta .
\end{aligned}
$$

Therefore,

$$
t_{i}\left(w_{-i}(\cdot), w_{i}(\cdot)\right)+\tilde{w}_{i}^{\prime}\left(K^{*}\right)>t_{i}\left(w_{-i}(\cdot), \tilde{w}_{i}^{\prime}(\cdot)\right)+\tilde{w}_{i}^{\prime}\left(K^{* \prime}\right) .
$$

Hence, when $v_{i}(\cdot) \equiv \tilde{w}_{i}^{\prime}(\cdot)$, the announcement of $w_{i}(\cdot)$ will be superior which contradicts the fact that the mechanism is s.i.i.c.

Q.E.D.

COROLlARY 3: If announced valuation functions are restricted to the class of continuous functions, ${ }^{4}$ the family of Groves mechanisms is identical to the set of all s.i.i.c. direct revelation mechanisms.

Proof: It is only required to show that $\tilde{w}_{i}^{\prime}(\cdot)$ in the proof of Theorem 3 can be chosen continuous and so that (i) $K^{* \prime}$ maximizes $\tilde{w}_{i}^{\prime}(\cdot)+\Sigma w_{-i}(\cdot)$ over $K$, (ii) $\tilde{w}_{i}^{\prime}\left(K^{*}\right)=-\Sigma w_{-i}\left(K^{*}\right)$, and (iii) $\tilde{w}_{i}^{\prime}\left(K^{* \prime}\right)=-\Sigma w_{-i}\left(K^{* \prime}\right)+\delta$.

\footnotetext{
${ }^{4}$ The public project under consideration is part of the larger economic system. If individuals recognize this, they may also evaluate various levels of the public project in the light of their predictions about equilibrium prices that would arise in these cases. It is known that the set of equilibria behaves only upper semi-continuously as the parameters are varied; and in this case we can treat the social decision about the public projects as a parameter of the private goods equilibrium. Due to potential discontinuities in the set of equilibria, we may expect corresponding discontinuities in willingnesses to pay for marginal units of the project, even if there are no income effects on the demand for the project itself.
} 
Let $\eta>0$ be such that $\eta<\left|K^{*}-K^{* \prime}\right|$ and

$$
\begin{aligned}
\tilde{w}_{i}^{\prime}(K) & =-\sum w_{-i}(K) \text { for } K \notin\left[K^{* \prime}-\eta, K^{* \prime}+\eta\right], \\
& =-\sum w_{-i}(K)+\delta\left(1-\frac{\left|K-K^{* \prime}\right|}{\eta}\right) \text { for } K \in\left[K^{* \prime}-\eta, K^{* \prime}+\eta\right] .
\end{aligned}
$$

By construction $\tilde{w}_{i}^{\prime}(\cdot)$ is continuous since $w_{j}(\cdot), j \neq i$, are continuous and satisfy (ii) and (iii). Moreover, it is easy to see that (i) is also satisfied since we always have

$$
\tilde{w}_{i}^{\prime}(K)+\Sigma w_{-i}(K)<\delta \text { for } K \neq K^{* \prime} .
$$

Q.E.D.

THEOREM 4: $A$ successful s.i.i.c. normalized revelation mechanism is a normalized Groves mechanism.

PROOF: Since the revelation mechanism is s.i.i.c. and normalized, agents will answer their true valuation function $v_{i}(\cdot), i=1, \ldots, N$, by Corollary 2 . Since it is successful, we can say that the decision is taken by maximizing the sum of the answers. Therefore it is a direct revelation mechanism. Hence, the result by Theorem 3.

Q.E.D.

We now extend the characterization to satisfactory mechanisms.

THEOREM 5: A satisfactory mechanism $(S, f)$ which satisfies the property of uniqueness of dominant strategies is such that there exist

$$
\psi_{i}, i=1, \ldots, N, \text { where } \psi_{i}: S_{i}^{\prime} \rightarrow V_{i}
$$

and a-normalized Groves mechanism, $N G M=\{V, g\}$ such that

$$
f(s)=g\left[\psi_{1}\left(s_{1}\right), \ldots, \psi_{N}\left(s_{N}\right)\right] .
$$

ProOF: Let $\mathscr{D}_{i}\left(v_{i}(\cdot)\right)$ be the unique dominant strategy of agent $i$ when the truth is $v_{i}(\cdot), i=1, \ldots, N$.

From the mechanism $(S, f)$, we construct a normalized revelation mechanism $(V, \phi)$ as follows: Let $\phi(w(\cdot))=f[\mathscr{D}(w(\cdot))]$ for any $w(\cdot) \in V$ where $V=\pi V_{i}$, the space of u.s.c. normalized valuation functions. The revelation mechanism $(V, \phi)$ is well-defined since $\mathscr{D}_{i}\left(v_{i}(\cdot)\right)$ is a singleton for $i=1, \ldots, N$.

We want to show that $(V, \phi)$ is s.i.i.c. Suppose it is not. For some $v_{i}(\cdot), w_{-i}(\cdot)$, there exists $w_{i}(\cdot) \neq v_{i}(\cdot)$ with $\phi\left(w_{-i}(\cdot), w_{i}(\cdot)\right)$ preferred by individual $i$ to $\phi\left(w_{-i}(\cdot), v_{i}(\cdot)\right)$, i.e., $f\left[\mathscr{D}_{-i}\left(w_{-i}(\cdot)\right), \mathscr{D}_{i}\left(w_{i}(\cdot)\right)\right]$ preferred to $f\left(\mathscr{D}_{-i}\left(w_{-i}(\cdot)\right)\right.$, $\left.\mathscr{D}_{i}\left(v_{i}(\cdot)\right)\right]$ where

$$
\begin{array}{r}
\mathscr{D}_{-i}\left(w_{-i}(\cdot)\right)=\left[\mathscr{D}_{1}\left(w_{1}(\cdot)\right), \ldots, \mathscr{D}_{i-1}\left(w_{i-1}(\cdot)\right), \mathscr{D}_{i+1}\left(w_{i+1}(\cdot)\right), \ldots,\right. \\
\left.\mathscr{D}_{N}\left(w_{N}(\cdot)\right)\right] ;
\end{array}
$$

therefore,

$$
u_{i}\left(\mathscr{D}_{-i}\left(w_{-i}(\cdot)\right), \mathscr{D}_{i}\left(w_{i}(\cdot)\right) ;\{S, f\}\right)>u_{i}\left(\mathscr{D}_{-i}\left(w_{-i}(\cdot)\right), \mathscr{D}_{i}\left(v_{i}(\cdot)\right) ;\{S, f\}\right)
$$

which contradicts the fact that $\mathscr{D}_{i}\left(v_{i}(\cdot)\right)$ is a dominant strategy. Also $(V, \phi)$ is 
successful since it has the same outcomes as $(S, f)$. Therefore, it is a successful s.i.i.c. normalized revelation mechanism and, consequently, a normalized Groves mechanism from Theorem 4.

Finally, to show that $\mathscr{D}_{i}^{-1}(\cdot), i=1, \ldots, N$, exist as functions we prove that $\mathscr{D}_{i}$ is univalent for $i=1, \ldots, N$. Suppose $\mathscr{D}_{i}$ is not univalent. Then, there exist $v_{i}(\cdot)$ and $v_{i}^{\prime}(\cdot)$ in $V_{i}$, with $v_{i}(\cdot) \neq v_{i}^{\prime}(\cdot)$ such that $\mathscr{D}_{i}\left(v_{i}(\cdot)\right)=\mathscr{D}_{i}\left(v_{i}^{\prime}(\cdot)\right)$. By definition $v_{i}(0)=v_{i}^{\prime}(0)=0$, but since $v_{i}(\cdot) \neq v_{i}^{\prime}(\cdot)$, there exists $K \in \mathscr{K}$, different from zero, such that $v_{i}(K) \neq v_{i}^{\prime}(K)$.

Without loss of generality, let $K^{*}$ be such that

$$
v_{i}\left(K^{*}\right)-v_{i}^{\prime}\left(K^{*}\right)=\varepsilon .
$$

Let $A=\sup \left[\sup _{K \in \mathscr{K}} v_{i}(K), \sup _{K \in \mathscr{K}} v_{i}^{\prime}(K)\right]$.

We can choose $v_{-i}(\cdot)$ such that:

$$
\begin{aligned}
& \sum v_{-i}\left(K^{*}\right)=-v_{i}^{\prime}\left(K^{*}\right)-\frac{\varepsilon}{2}, \\
& \sum v_{-i}(0)=0, \quad \text { and } \\
& \sum v_{-i}(K)=-A-\varepsilon .
\end{aligned}
$$

Then, clearly,

and

$$
\sum v_{-i}\left(K^{*}\right)+v_{i}\left(K^{*}\right)>\sum v_{-i}(K)+v_{i}(K) \quad \forall K \in \mathscr{K}, \quad K \neq K^{*},
$$

$$
\sum v_{-i}(0)+v_{i}^{\prime}(0)>\sum v_{-i}(K)+v_{i}^{\prime}(K) \quad \forall K \in \mathscr{K}, \quad K \neq 0 .
$$

Therefore, we are able to construct $\left(v_{i}(\cdot)\right)$ such that $\left[v_{-i}(\cdot), v_{i}(\cdot)\right]$ and $\left[v_{-i}(\cdot), v_{i}^{\prime}(\cdot)\right]$ should lead to different decisions under the Pareto criterion, and they do not, contradicting the successfulness of $(V, \phi)$.

Therefore, $\mathscr{D}_{i}(\cdot)$ is univalent for $i=1, \ldots, N$. If we define $\psi_{i}=\mathscr{D}_{i}^{-1}(\cdot)$ from $S_{i}^{\prime}$ into $V_{i}$, then,

$$
g\left[\psi_{1}\left(s_{1}\right), \ldots, \psi_{N}\left(s_{N}\right)\right]=f\left[\mathscr{D}_{1} \cdot \mathscr{D}_{1}^{-1}\left(s_{1}\right), \ldots, \mathscr{D}_{N} \cdot \mathscr{D}_{N}^{-1}\left(s_{N}\right)\right]=f(s) .
$$

Q.E.D.

If the assumption of uniqueness in Theorem 5 is left out, it is only possible to obtain a weaker characterization. Indeed, consider the following example.

Let $\mathscr{K}=\{0,1\}, S_{i}=R^{2}, s_{i}=\left(s_{i 1}, s_{i 2}\right), i=1, \ldots, N$, and define the mechanism as follows:

$$
\begin{aligned}
d(s) & =1 \quad \text { if } \quad \sum s_{i 1} \geqslant 0, \\
& =0 \quad \text { if } \quad \sum s_{i 1}<0, \\
t_{i}(s) & =\sum s_{-i 1}+\sum s_{-i 2} \text { if } d(s)=1, \\
& =\sum s_{-i 2} \text { if } d(s)=0, \text { for } i=1, \ldots, N .
\end{aligned}
$$

Then, the set of dominant strategies is:

$$
\mathscr{D}_{i}\left(v_{i}(\cdot)\right)=\left\{\left(s_{i 1}, s_{i 2}\right) \in R^{2} \mid s_{i 1}=v_{i}(1)\right\} \text {. }
$$


Therefore, this mechanism cannot be expressed as $g[\psi(s)]$ for any Groves mechanism since $t_{i}(s), i=1, \ldots, N$ are not constant over $\Pi_{i=1}^{N} \mathscr{D}_{i}\left(v_{i}(\cdot)\right)$.

However, all satisfactory mechanisms can be shown to be isomorphic to extended Groves mechanisms.

THEOREM 6: Let $(S, f)$ be a satisfactory mechanism. Then, there exist functions $\psi_{i}$ from $S_{i}^{\prime}$ into $V_{i}, i=1, \ldots, N$, and an extended normalized Groves mechanism $(V, G)$ such that:

$$
\bigcup_{s^{\prime} \in S(s)} f\left(s^{\prime}\right)=G\left[\psi_{1}\left(s_{1}\right), \ldots, \psi_{N}\left(s_{N}\right)\right]
$$

where $S(s)=\left\{s^{\prime} \mid s\right.$ and $s^{\prime}$ belong to the same $\left.\mathscr{D}(w(\cdot))\right\}$.

Proof: It is a matter of routine to check that Theorem 2, Theorem 3, and Theorem 4 are true for extended (direct) revelation mechanisms. The proof then follows the lines of the proof of Theorem 5 with some differences noted below. Now, $\mathscr{D}_{i}\left(v_{i}(\cdot)\right)$ is the set of dominant strategies of agent $i$ when the truth is $v_{i}(\cdot)$, $i=1, \ldots, N$. We construct an extended normalized revelation mechanism as follows. Let $V_{i}$ be the set of normalized u.s.c. valuation functions and let

$$
\Phi(w(\cdot))=f[\mathscr{D}(w(\cdot))] \quad \forall w(\cdot) \in V .
$$

$\Phi$ is now a correspondence with two properties: $s^{1} \in \mathscr{D}(v(\cdot))$ and $s^{2} \in \mathscr{D}(v(\cdot))$ implies that both $d\left(s^{1}\right)$ and $d\left(s^{2}\right)$ maximize $\Sigma v_{i}(\cdot)$, otherwise the mechanisms would not be successful.

Also, $v_{i}\left(d\left(s^{1}\right)\right)+T_{i}\left(s^{1}\right)=v_{i}\left(d\left(s^{2}\right)\right)+T_{i}\left(s^{2}\right)$.

Otherwise there would exist $t_{i}(\cdot) \in T_{i}(\cdot)$ such that, without loss of generality:

$$
v_{i}\left(d\left(s^{1}\right)\right)+t_{i}\left(s^{1}\right)>v_{i}\left(d\left(s^{2}\right)\right)+t_{i}\left(s^{2}\right)
$$

and then $s_{i}^{2}$ would not be a dominant strategy for agent $i$.

As in Theorem 5, it is shown that $(V, \Phi)$ is s.i.i.c. By Theorem $4,(V, \Phi)$ is then an extended normalized Groves mechanism.

Now, if $v_{i}(\cdot) \neq v_{i}^{\prime}(\cdot)$ then $\mathscr{D}_{i}\left(v_{i}(\cdot)\right) \cap \mathscr{D}_{i}\left(v_{i}^{\prime}(\cdot)\right)=\varnothing$. Suppose, on the contrary, that there exists $s_{i} \in \mathscr{D}_{i}\left(v_{i}(\cdot)\right) \cap \mathscr{D}_{i}\left(v_{i}^{\prime}(\cdot)\right)$. Then, $v_{i}(\cdot)$ and $v_{i}^{\prime}(\cdot)$ may lead to the same project $K^{*}$. As in Theorem 5 , we can choose $\Sigma w_{-i}(\cdot)$ such that $v_{i}(\cdot)$ and $v_{i}^{\prime}(\cdot)$ should lead to different projects, contradicting the successfulness of $(V, \Phi)$.

It is therefore possible to define the function $\psi_{i}(\cdot)=\mathscr{D}_{i}^{-1}(\cdot), i=1, \ldots, N$, which is such that: $\mathscr{D}_{i} \cdot \mathscr{D}_{i}^{-1}\left(s_{i}\right)=S_{i}\left(s_{i}\right)=\left\{s_{i}^{\prime} \mid s_{i}\right.$ and $s_{i}^{\prime}$ belong to the same $\mathscr{D}_{i}\left(v_{i}(\cdot)\right\}$. Then,

$$
\begin{aligned}
\Phi\left[\psi_{1}\left(s_{1}\right), \ldots, \psi_{N}\left(s_{N}\right)\right] & =f\left[\mathscr{D}_{1} \cdot \mathscr{D}_{1}^{-1}\left(s_{1}\right), \ldots, \mathscr{D}_{N} \cdot \mathscr{D}_{N}^{-1}\left(s_{N}\right)\right] \\
& =\bigcup_{s^{\prime} \in S(s)} f(s) .
\end{aligned}
$$

\section{Harvara University and}

Ecole Polytechnique 


\section{APPENDIX}

We show the non-existence of successful s.i.i.c. direct revelation mechanisms for the fixed-size unique public project case when preferences are not separable. Now, in defining normalized revelation mechanisms, the question is: What is your utility function normalized in such a way that the utility of "no project and transfer $t$ " equals the transfer $t$. For a separable utility function we had:

$$
\begin{aligned}
& u_{i}\left(0, t_{i}\right)=t_{i}, \\
& u_{i}\left(1, t_{i}\right)=t_{i}+v_{i}, \quad v_{i} \in R .
\end{aligned}
$$

An example of a nonseparable normalized utility function is:

$$
\begin{aligned}
& u_{i}\left(0, t_{i}\right)=t_{i}, \\
& u_{i}\left(1, t_{i}\right)=t_{i}+v_{i}\left(t_{i}\right), \quad v_{i}(\cdot) \text { u.s.c. on } R .
\end{aligned}
$$

For the separable case, the evaluation of the project is a constant; it is a function of the transfer for the nonseparable case.

Finally, when utility functions are nonseparable, we must change slightly the notions of successfulness and incentive compatibility. We say that a $D R M$ is successful if for any transfer program $\left(t_{1}, \ldots, t_{N}\right), f(w(\cdot))$ maximizes $\Sigma_{i=1}^{N} u_{i}(w(\cdot) ; D R M)$. We say that an $R M$ is s.i.i.c. if the true normalized utility function is a dominant strategy. We can now formulate the following theorem:

THEOREM 7: If the space of allowable evaluations of the project includes all constant functions (of the transfer), and the step functions with values $a$ and $b, b>a$, for transfers above and below the level $x$, then there exists no successful normalized s.i.i.c. revelation mechanism.

PROOF: We prove the theorem by providing a counterexample. Consider the following two agents: Agent 1 is such that

$$
\begin{aligned}
& u_{1}\left(0, t_{1}\right)=t_{1}, \\
& u_{1}\left(1, t_{1}\right)=t_{1}+c .
\end{aligned}
$$

He has a separable utility function. Agent 2 is such that

$$
\begin{aligned}
u_{2}\left(0, t_{2}\right) & =t_{2}, \\
u_{2}\left(1, t_{2}\right) & =t_{2}+a \quad \text { if } \quad t_{2}<x, \\
& =t_{2}+b \quad \text { if } \quad t_{2} \geqslant x, \quad \text { with } a<b .
\end{aligned}
$$

Agent 2's evaluation of the project increases when the transfer he receives reaches a threshold value. Here the $D R M$ associates to a value $(c, a, b, x)$ a vector $\left(d, t_{1}, t_{2}\right) \in\{0,1\} \times R \times R$, where $d$ is the decision and $\left(t_{1}, t_{2}\right)$ the transfers received.

Let us consider a particular combination $(a, b, c)$ which remains fixed for the rest of the argument and such that: $a+c<0, b+c>0, c<0$, and let $x$ vary.

Successfulness means here that

$$
\begin{array}{llll}
\text { if } t_{2}<x & \text { then } d=0 & \text { since } a+c<0, & \text { and } \\
\text { if } t_{2} \geqslant x & \text { then } d=1 & \text { since } b+c>0 .
\end{array}
$$

We prove that there exists no successful s.i.i.c. $D R M$ by contradiction in a sequence of lemmata.

LEMMA 1: It is not true that the project is accepted for all $x$.

PROOF: Whenever the project is accepted $t_{2}>x$ by successfulness. Then, there would be an incentive to set $x$ high to force a high $t_{2}$ (since $u_{2}\left(1, t_{2}\right)$ is increasing in $\left.t_{2}\right)$, contradicting s.i.i.c.

Q.E.D.

\section{LEMMA 2: It is not true that the project is rejected for all $x$.}

PROOF: If so, the transfer to Agent 2 would be given by a function $t_{2}(x)$ which would be everywhere below $x$. Clearly, no constant function has this property. Let $x^{\prime}$ and $x^{\prime \prime}$ be such that $t_{2}\left(x^{\prime}\right)<t_{2}\left(x^{\prime \prime}\right)$; then if the true tastes are at $x^{\prime}$, the individual has an incentive to use $x^{\prime \prime}$ instead (since $u_{2}\left(0, t_{2}\right)$ is increasing in $\left.t_{2}\right)$.

Q.E.D. 
Let $X$ be the set of $x$ that lead to acceptance and $X^{\prime}$ be the complement of that set.

\section{LEMMA 3: The transfers to Individual 2 must be constant on $X$ and $X^{\prime}$.}

PROOF: If not (say on $X$ ), then if the true $x_{0} \in X$ were associated with a lower transfer than some other $x_{1} \in X$, the statement $x_{1}$ would be better than $x_{0}$ since the transfer would be higher and, since $u_{2}\left(1, t_{2}\right)$ is increasing in $t_{2}$, contradicting s.i.i.c.

In the case of $X^{\prime}$ the same argument holds.

Q.E.D.

Take $x \in X$ and $x^{\prime} \in X^{\prime}$.

LEMMA 4: $t_{2}(x)=t_{2}\left(x^{\prime}\right)+c$.

PROOF: First, let us compare the transfer with the statement $(a, b, x)$ to the transfer with the constant statement $e$, such that $e \geqslant-c$. These transfers must be equal for, if they were higher at $(a, b, x)$, then $(a, b, x)$ would be answered instead of $e$ when $e$ was true, and vice versa (since $u_{2}\left(1, t_{2}\right)$ is increasing).

By a similar argument, we note that the transfer at $\left(a, b, x^{\prime}\right)$ must equal that at constant statements $e^{\prime}$ such that $e^{\prime}<-c$.

Then, by Theorem 2 and Lemma 1 (Section 2), we know that transfer at $e \geqslant-c$ is equal to that at $e^{\prime}<-c$ plus $c$.

Q.E.D.

We are now in a position to prove Theorem 7.

ProOF OF THEOREM 7: By the above lemmata, we know that there exists a number $\bar{t}$ such that the transfer to Agent 2 throughout the region $X$ is $\bar{t}+c$ and the project is accepted, and throughout $X^{\prime}$ it is $\bar{t}$ and the project is rejected. Moreover, since $c<0, \bar{t}+c<\bar{t}$. Since the mechanism is successful, $x \in X$ implies that $x \leqslant \bar{t}+c$ for if $x>\bar{t}+c$, then the preferences at $\bar{t}+c$ would lead to rejection rather than acceptance. Likewise, $x^{\prime} \in X^{\prime}$ implies $x^{\prime}>\bar{t}$.

Therefore, letting $\bar{x} \in(\bar{t}+c, \bar{t})$, we obtain a contradiction to either $\bar{x} \in X$ or $\bar{x} \in X^{\prime}$, and hence we contradict the fact that the mechanism produces a well-defined outcome.

Q.E.D.

REMARK: It is clear from the above proof that the constancy of the evaluations on the sets $\{t / t \geqslant x\}$ and $\{t / t<x\}$ is not necessary to obtain the counterexample. The crucial feature is that $u_{2}\left(1, t_{2}\right)=$ $t_{2}+v_{2}\left(t_{2}\right)$ is increasing in $t_{2}$.

\section{COROLLARY 4: Under the conditions of Theorem 7, there exists no satisfactory mechanism.}

PROOF: Note first that from an extended normalized revelation mechanism one can always select a normalized revelation mechanism. Suppose, then, that there exists a satisfactory mechanism. From the first part of Theorem 6 and the above remark, there exists a normalized successful s.i.i.c. revelation mechanism, a contradiction in view of Theorem 7 .

\section{REFERENCES}

[1] Clarke, E. H.: "Multipart Pricing of Public Goods,” Public Choice, 8 (1971), 19-33.

[2] Gibbard, A.: "Manipulation of Voting Schemes: A General Result," Econometrica, 41 (1973), 587-602.

[3] Groves, T.: "Incentives in Teams,” Econometrica, 41 (1973), 617-631.

[4] — : "Information, Incentives and the Internalization of Production Externalities," Discussion Paper No. 87, The Center for Mathematical Studies in Economics and Management Science, Northwestern University, 1974.

[5] Groves, T., AND M. Loeb: "Incentives and Public Inputs," Journal of Public Economics, 4 (1975), 211-226.

[6] Satterthwaite, M. A.: "Strategy-Proofness and Arrow's Conditions: Existence and Correspondence Theorems for Voting Procedures and Social Welfare Functions," Journal of Economic Theory, 10 (1975), 187-217.

[7] Vickrey, W.: "Counterspeculation, Auctions and Competitive Sealed Tenders," Journal of Finance, 16 (1961), 8-37. 